\title{
WNT5A is a Key Regulator of the Epithelial- Mesenchymal Transition and Cancer Stem Cell Properties in Human Gastric Carcinoma Cells
}

\author{
Maki Kanzawa ${ }^{a}$ b Shuho Semba ${ }^{a}$ Shigeo Hara ${ }^{b}$ Tomoo Itoh $^{b}$ \\ Hiroshi Yokozaki ${ }^{a}$ \\ Divisions of a Pathology and ${ }^{b}$ Diagnostic Pathology, Department of Pathology, Kobe University Graduate School \\ of Medicine, Kobe, Japan
}

\section{Key Words}

WNT5A - Gastric carcinoma · Epithelial-mesenchymal transition · Cancer stem cells · SNAI1 · CD133

\begin{abstract}
Objective: Direct interaction with cancer-associated fibroblasts triggers WNT5A expression in human gastric carcinoma (GC) cells. In this study, we performed gene transduction experiments to investigate the significance of WNT5A in the GC tumor microenvironment. Methods: Gene transduction ( $p$ WNT5A and shWNT5A) was performed in human GC-derived MKN-7 cells. Altered gene expression was examined by RT-PCR and CDNA microarray analysis. Immunohistochemical examination was carried out in human GC tissues. Results: Transduction of exogenous WNT5A expression into MKN-7 cells upregulated genes related to the epithelial-mesenchymal transition (EMT) and cancer stem cells (CSCs), and the pWNT5A transfectant showed high tumorigenicity in vivo. These results were confirmed by knockdown experiments using a lentivirus expressing shWNT5A. A cDNA microarray analysis suggested that depletion of endogenous WNT5A downregulated genes involved in intracellular signaling, chemokine-cytokine interaction and focal adhesion. High levels of WNT5A expression were observed in $66 \%$ of GC cases, with significant correla-
\end{abstract}

tion with histological type. Interestingly, in intestinal-type GCs, WNT5A expression was detected in the periphery of tumor nests. Conclusions: WNT5A regulates the induction of EMT and the maintenance of CSC properties in MKN-7 cells. WNT5A may play an important role in constructing an advantageous tumor microenvironment for the progression and development of human GC.

Copyright $\odot 2013$ S. Karger AG, Basel

\section{Introduction}

Stroma contributes significantly to the genesis and progression of a variety of human malignancies by stimulating cell growth, invasiveness and angiogenesis. Such a tumor microenvironment, also referred to as a cancer niche, consists of many cellular components, including fibroblasts, myofibroblasts, immune cells and blood vessels, and acellular components, including extracellular matrix, cytokines and growth factors. Cancer-associated fibroblasts (CAFs) are believed to enhance the tumorigenic potential of epithelial cells $[1,2]$.

In our previous study examining the biological effects of CAFs on the aggressiveness of human gastric carcinoma (GC) cells, we discovered the importance of direct cell-cell contact between GC cells and CAFs to promote

\section{KARGER}

E-Mail karger@karger.com

www.karger.com/pat
(C) 2013 S. Karger AG, Basel

1015-2008/13/0805-0235\$38.00/0
Shuho Semba, MD, PhD

Division of Pathology, Department of Pathology Kobe University Graduate School of Medicine

7-5-1 Kusunoki-cho, Chuo-ku, Kobe 650-0017 (Japan)

E-Mail semba@med.kobe-u.ac.jp 
induction of the epithelial-mesenchymal transition (EMT) and restoration of cancer stem cell (CSC) properties. In an experimental model of coculturing of human GC-derived MKN-7 cells and bone marrow-derived CAFs, their direct interaction increased WNT5A and transforming growth factor (TGF) $\beta$ expression in GC cells and CAFs, respectively [3]. WNT5A is a member of the WNT family of secreted growth factors, which are 38$45 \mathrm{kDa}$ rich with cysteine and hydrophobic signal peptides and play crucial roles in various developmental processes and tissue homeostasis in adults $[4,5]$. In human GC cells, WNT5A activated focal adhesion kinase and a small GTP-binding protein, Rac, and subsequently caused cell migration and membrane ruffling [6]. In addition, WNT5A cooperates with Ror2, a receptor tyrosine kinase, and accelerates filopodia formation and cell migration by activating the noncanonical WNT-JNK pathway and inhibiting the canonical WNT- $\beta$-catenin pathway [4]. Constitutive activation of WNT5A and Ror2 dramatically enhanced the invasiveness of human osteosarcoma cell lines SaOS-2 and U2OS [7]. These results indicate the possible function of WNT5A in promoting the invasiveness of human malignancies; indeed, knockdown of WNT5A in GC cells reduced the number of liver metastases that formed in mice [8]. In human GC tissues, overexpression of WNT5A has been reported in $30 \%$ of GC cases, with statistical correlation with tumor size, the incidence of lymph node metastasis and high clinicopathological stage [6]. Furthermore, in our previous study, induction of WNT5A stimulated by contact with CAFs restored CD133 CSC marker levels as well as treatment with recombinant WNT5A [3]. Supporting this WNT5A function, a recent study has revealed that WNT5A enhances Notch1 intracellular domain transcription activity [9], which is one of the key regulators of EMT and the CSC phenotype during the development and progression of tumors $[10,11]$.

The CSC model, also referred to as the hierarchical model, is the concept that only CSCs can generate a tumor, based on their self-renewal properties and enormous proliferative potential through their activation or progenitor production [16]. With new insights into the cellular mechanism leading to cancer, the metastatic potential of tumor cells is thought to be a reflection of the ability of CSCs to clonally initiate tumorigenicity at distant sites $[17,18]$. Therefore, understanding CSCs is an urgent problem not only for cancer prevention but also for the development of novel therapeutic approaches. CD133 is a common CSC marker in a large variety of human malignancies, including those of the brain, co- lon, liver, pancreas and prostate [15-19]. CD133 is a 5 -transmembrane-domain glycoprotein, originally identified as a cell surface antigen present on CD34+ hematopoietic stem cells [28], and the antibody against the CD133 epitope is now therefore exploited to distinguish CSCs that compose a minor population within a tumor mass [29].

In the present study, we attempted to investigate the role of WNT5A in the acquisition of malignant potential by MKN-7 cells. Induction and knockdown of WNT5A was performed to investigate the biological significance of WNT5A, particularly targeting the induction of EMT and the restoration of CSC properties. To gain further insight into the biological significance of WNT5A in human GC cells, we also surveyed gene expression patterns of MKN-7 cells in the presence or absence of knockdown of WNT5A using a cDNA microarray. These investigations were followed by the immunohistochemical examination of human GC tissues, and the correlations with clinicopathological findings were analyzed.

\section{Materials and Methods}

\section{Cell Lines and Tissue Samples}

Human GC-derived MKN-7 and MKN-74 cells were gifts from Prof. Toshiyuki Suzuki (Fukushima Medical University, Fukushima, Japan) [22, 23]. HSC-45 and HSC-57 cells were established by Kazuyoshi Yanagihara (Yasuda Women's University, Hiroshima, Japan) $[14,15]$. TMK-1 cells were established from poorly differentiated adenocarcinoma of the stomach. Cells were cultured in RPMI-1640 medium with 10\% fetal bovine serum. For the inhibition of WNT signaling, XAV939 was purchased from Sigma (St. Louis, Mo., USA). A total of 42 cases of various histological subtypes of human GC tissues surgically removed at Kobe University Hospital (Kobe, Japan) were employed. Twenty-one cases had metastases to the regional lymph nodes, while the other 21 cases had no metastasis, and each of the cases invaded to the muscularis propria, at least. Informed consent was obtained from all patients, and the study was approved by the institutional review committee of Kobe University.

\section{Gene Transduction}

Human WNT5A-expressing vector (pWNT5A) was kindly provided by Prof. Akira Kikuchi (Osaka University, Osaka, Japan). pWNT5A [6] was transfected into MKN-7 cells using XtremeGENE HP DNA Transfection Reagent (Roche, Basel, Switzerland) following the manufacturer's protocol. For the suppression of endogenous WNT5A expression, we used a lentiviral shRNA transduction system, the WNT5A targeting MISSION shRNA (shWNT5A) lentiviral particles (Sigma). Briefly, $24 \mathrm{~h}$ before infection, MKN-7 cells were plated in a $10-\mathrm{cm}$ dish at $45-50 \%$ confluency. The following day, hexadimethrine bromide (Sigma), a transduction enhancer, was added to the well at a concentration of $8 \mu \mathrm{g} / \mathrm{ml}$, and viral particles were removed and replaced with fresh medium containing $1 \mu \mathrm{g} / \mathrm{ml}$ puromycin. Cells were grown to ap- 
proximately $90 \%$ confluency and subcultured in medium containing puromycin. Before lentiviral infection, a puromycin titration was performed to identify the minimum concentration of puromycin that caused complete cell death of MKN-7 cells after 3 days.

\section{RT-PCR and Quantitative Real-Time RT-PCR}

RT-PCR was performed using the following primer sets: $W N$ T5A, $5^{\prime}$-ATT CAC ATC CCC TCA GTT GC- $3^{\prime} / 5^{\prime}$-CCA TTT GGA ATA AGC CTT TCG-3', and GAPDH, $5^{\prime}$-TCC ACT GGC GTC TTC ACC- $3^{\prime} / 5^{\prime}$-GGC AGA GAT GAT GAC CCT TTT- $3^{\prime}$. After an initial incubation at $50^{\circ} \mathrm{C}$ for $30 \mathrm{~min}$ and denaturation at $95^{\circ} \mathrm{C}$ for $15 \mathrm{~min}$, the following cycling conditions (40 cycles) were used for PCR: denaturation at $94^{\circ} \mathrm{C}$ for $15 \mathrm{~s}$, annealing at $60^{\circ} \mathrm{C}$ for $30 \mathrm{~s}$ and extension at $72^{\circ} \mathrm{C}$ for $1 \mathrm{~min}$. Quantitative real-time RT-PCR was performed using a QuantiTect SYBR Green PCR kit (Qiagen, Hilden, Germany) in triplicate. The primer sets used in the present study are as follows: Snail (SNAI1), 5'-GCC TGG GCG ACA AGA TTG A-3'/5'-CCA ACA GCT CCC AAT TCA C-3'; E-cadherin (CDH1), $5^{\prime}$-CGG GAA TGC AGT TGA GGA TC- $3^{\prime} / 5^{\prime}$-AGG ATG GTG TAA GCG ATG GC-3'; Vimentin (VIM); $5^{\prime}$-ACC AGG TCC GTG TCC TCG T-3 $3^{\prime} / 5^{\prime}$-CTG CCC AGG CTG TAG GTG-3'; CD133, $5^{\prime}$-CAG AGT ACA ACG CCA AAC CA-3' $/ 5^{\prime}$-CCC CAG GAC ACA GCA TAG AA- ${ }^{\prime}$; CD44, 5' ${ }^{\prime}$-TGA GAC TTG CTG GCC TCT CCG T- $3^{\prime} / 5^{\prime}$-TGA GACTTG CTG GCT CTCCGT- ${ }^{\prime}$; TGF- $\beta 1$ (TGFBI), $5^{\prime}$-ACT GCA AGT GGA CAT CAA CG- $3^{\prime} / 5^{\prime}$-TGC GGA AGT CAA TGT ACA GC- $3^{\prime}$; $M M P 2,5^{\prime}$-ACG ACC GCG ACA AGA AGT AT- $3^{\prime} / 5^{\prime}$-ATT TGT TGC CCA GGA AAG TG-3' ${ }^{\prime}$, and $M M P 9$, $5^{\prime}$-GAC AAG CTC TTC GGC TTG TG-3' $/ 5^{\prime}$-TCG CTG GTA CAG GTC GAG TA- $3^{\prime}$. The threshold cycle $\left(\mathrm{C}_{\mathrm{T}}\right)$ values were determined by plotting the observed fluorescence against the cycle number. Each $\mathrm{C}_{\mathrm{T}}$ value was analyzed using the comparative threshold cycle method and normalized to the $\mathrm{C}_{\mathrm{T}}$ values of GAPDH. The relative gene expression levels were estimated using the following formula:

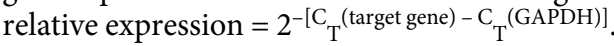

\section{Western Blotting}

Cells were lysed in a lysis buffer $(50 \mu \mathrm{M}$ Tris-HCl, $\mathrm{pH} 7.4,125$ $\mu \mathrm{M} \mathrm{NaCl}, 0.1 \%$ Triton $\mathrm{X}$ and $5 \mu \mathrm{M}$ ethylenediaminetetraacetic acid). Proteins were separated by Running Buffer Solution (Wako, Osaka, Japan) onto a 5-20\% polyacrylamide gel (SuperSep, Wako) and then immunoblotted with antibodies against WNT5A (Abcam, Cambridge, Mass., USA), SNAI1 (Abcam), CDH1 (Sigma) and $\beta$-actin (Sigma). Horseradish peroxidase-conjugated donkey antirat IgG and sheep antimouse IgG (GE Healthcare, Piscataway, N.J., USA) were used as secondary antibodies. Proteins were visualized using enhanced chemiluminescence.

\section{Immunocytochemistry}

Cells in chamber slides were fixed with $4 \%$ formalin and incubated with blocking solution containing $1 \%$ bovine serum albumin in PBS. Antibodies against WNT5A (Abcam) were also used for immunocytochemistry. Cells were incubated with primary antibody for $2 \mathrm{~h}$ and then incubated with Cy2-conjugated anti-rabbit/mouse IgGs. Nuclei were stained with 4',6-diamidino-2-phenylindole.

\section{Cell Proliferation Assay and Cell Migration Assay}

Cells were seeded at a density of $1.0 \times 10^{5}$ onto a $10-\mathrm{cm}$ dish. The cell number was counted every $24 \mathrm{~h}$ for 4 days. Cell migration was assessed in the cell culture inserts (BD Falcon, Franklin Lakes, N.J., USA). In each tissue culture plate filled with the usu- al culture medium, we placed cell culture inserts and added MKN-7 cells and serum-free medium, then incubated for $96 \mathrm{~h}$. Thereafter, the medium was discarded, nonmigrated cells were removed with cotton and the membranes were cut out of the chamber, then fixed with $4 \%$ paraformaldehyde and stained with $0.5 \%$ hematoxylin. The motility was evaluated under a light microscope by counting the number of cells that had migrated into the membrane.

\section{Flow Cytometric Analysis}

To analyze cellular DNA content, cells sorted with magneticactivated cell sorting were fixed in $70 \%$ methanol, treated with RNase A and stained with propidium iodide. The analysis was performed with a fluorescence-activated cell sorting FACSCalibur cytometer (BD Biosciences, San Jose, Calif., USA). Experiments were repeated 3 times.

\section{Mouse Xenograft Model}

pWNT5A-transfected MKN-7 cells and control cells were inoculated into the right flank of severe combined immunodeficiency (SCID) mice (Japan Clea, Tokyo, Japan) with matrigel in a 1:1 ratio. Hematoxylin and eosin staining was carried out on paraffinembedded sections following standard protocols to confirm development of subcutaneous tumors. The initiating cell frequency was calculated using extreme limiting dilution analysis software. Extreme limiting dilution analysis is suitable for any limiting dilution problem, but special methods are implemented to give reliable results in extreme data situations, for example when all the assays give positive or negative results (http://bioinf.wehi.edu.au/ software/elda/).

\section{cDNA Microarray Analysis}

Total RNAs were extracted from the shWNT5A-infected MKN-7 and control cells using an RNeasy Mini Kit (Qiagen). The concentration of RNA was determined by measuring the absorbance at $260 \mathrm{~nm}$ in a spectrophotometer. A cDNA microarray was performed by Human Expression Array version 2.0 (Agilent, Palo Alto, Calif., USA). The in vitro transcription, oligonucleotide array hybridization and scanning were performed according to Takara Bio protocols (Takara Bio, Otsu, Japan). Briefly, double-stranded cDNA was synthesized from total RNA and labeled with an RNA Fluorescence Labeling Core Kit (Takara Bio). Arrays were then scanned with a GeneArray scanner (Agilent) to obtain image and signal intensities. After data normalization, significance analysis of microarray plot analysis was performed, and significantly altered genes were identified in accordance with the manufacturer's protocol (http://chem.agilent.com).

\section{Immunohistochemistry}

Tissue sections $(4 \mu \mathrm{m})$ of formalin-fixed and paraffin-embedded human GC were used. Sections were pretreated in a microwave oven in Target Retrieval Solution (Dako, Glostrup, Denmark) for $15 \mathrm{~min}$ to retrieve antigenicity. After peroxidase activity was blocked with $3 \% \mathrm{H}_{2} \mathrm{O}_{2}$ for $15 \mathrm{~min}$, the sections were incubated with $5 \%$ bovine serum albumin for $30 \mathrm{~min}$ at room temperature. Antibodies against WNT5A (Abcam) were incubated with tissue samples overnight at $4^{\circ} \mathrm{C}$ and detected by incubating for $30 \mathrm{~min}$ with biotinylated goat antirabbit immunoglobulins, and the signal was amplified and visualized using the substrate-chromogen solution. Immunoreactivity of WNT5A was graded according to the
WNT5A Regulates EMT and CSCs in Human Gastric Carcinoma Cells
Pathobiology 2013;80:235-244 DOI: $10.1159 / 000346843$ 


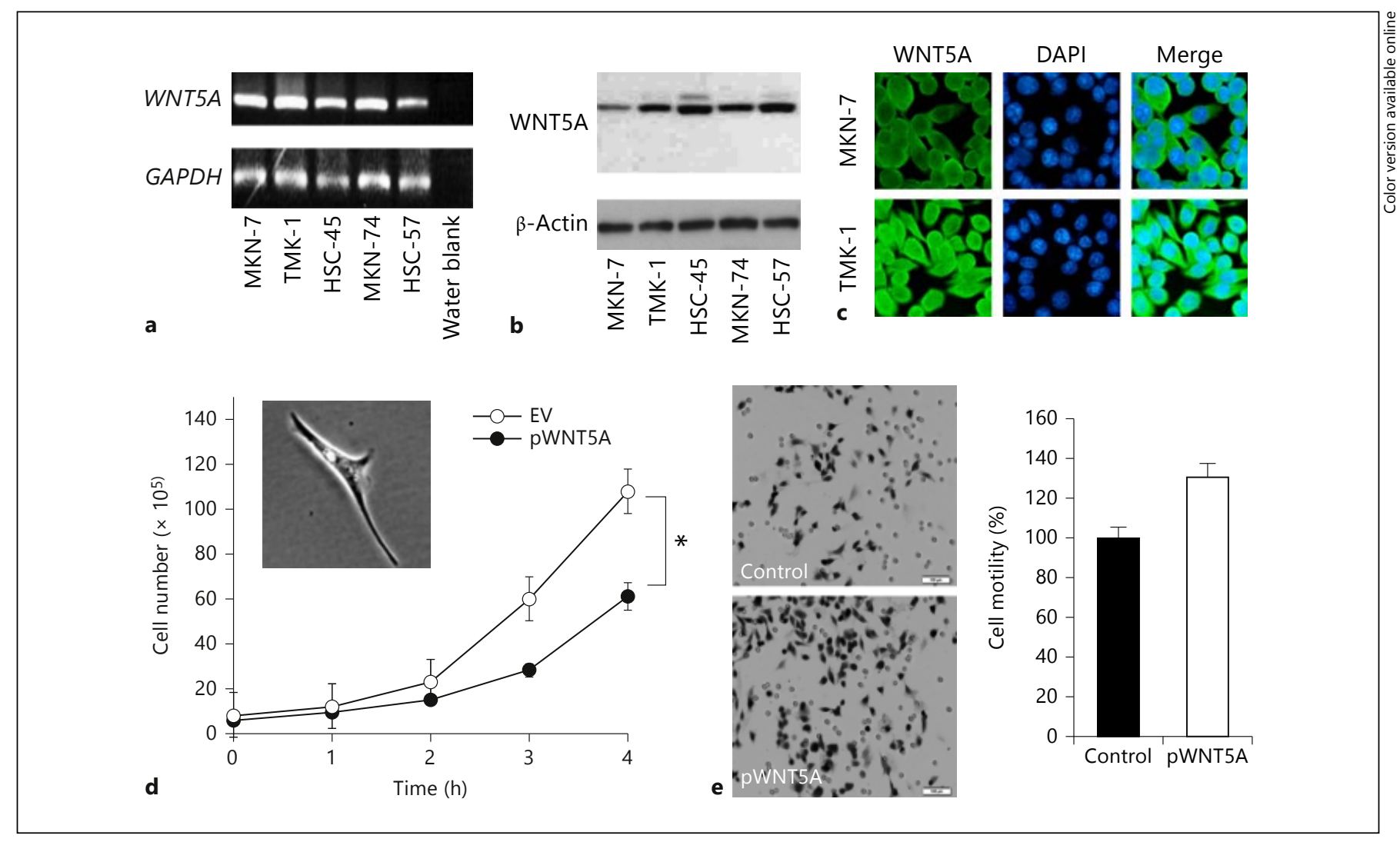

Fig. 1. Expression of WNT5A and the effects of transduction of pWNT5A in human GC-derived cells. a Expression of WNT5A transcripts in GC cell lines (RT-PCR). The mRNA levels of GAPDH expression were analyzed as a loading control. $\mathbf{b}$ Expression of WNT5A protein in GC cell lines (Western blot). $\beta$-Actin was used as a control. c Representative illustrations of WNT5A expression in

number of stained cells, and when over $50 \%$ of tumor cells showed moderate to intense immunoreactivity, we defined the case as having high levels of WNT5A expression.

\section{Results}

Transduction of WNT5A Does Not Increase Tumor Activity in GC Cells

We first examined endogenous expression of WNT5A in 5 human GC-derived cell lines (MKN-7, TMK-1, HSC-45, MKN-74 and HSC-57). Endogenous expression of WNT5A at both mRNA and protein levels was detected, which was also confirmed by immunofluorescence (fig. 1a-c). Since MKN-7 cells expressed low levels of WNT5A in comparison with the other cell lines, we chose MKN-7 cells for the subsequent gene transfection
MKN-7 and TMK-1 cells. Nuclei were stained with 4',6-diamidino2-phenylindole (DAPI). d Cell proliferation assay in MKN-7 cells after transfection of pWNT5A and EV. A representative illustration of the pWNT5A transfectant is shown in the upper left corner. e Cell motility assay. Left: representative illustration of migration of MKN-7 cells in the presence or absence of pWNT5A transfection.

experiments. To investigate the biological significance of WNT5A in human GC cells, we attempted to transfect pWNT5A into MKN-7 cells. Morphologically, the pWNT5A transfectant showed spindle-like changes and protruded; however, transduction of pWNT5A into MKN-7 cells suppressed cell growth in comparison with the mock control (fig. 1d). We also examined the impact of WNT5A on cell motility. The pWNT5A transfectant demonstrated slightly higher cell motility ( $\mathrm{p}<$ 0.05; fig. 1e).

\section{WNT5A Induces EMT and Restores CSC Properties}

Evidence has accumulated that WNT signaling plays an important role in the induction of EMT and the acquisition and maintenance of CSC characteristics $[18,19]$. We therefore investigated altered levels of gene expression of SNAI1, CDH1, VIM and CD133 in the presence or 


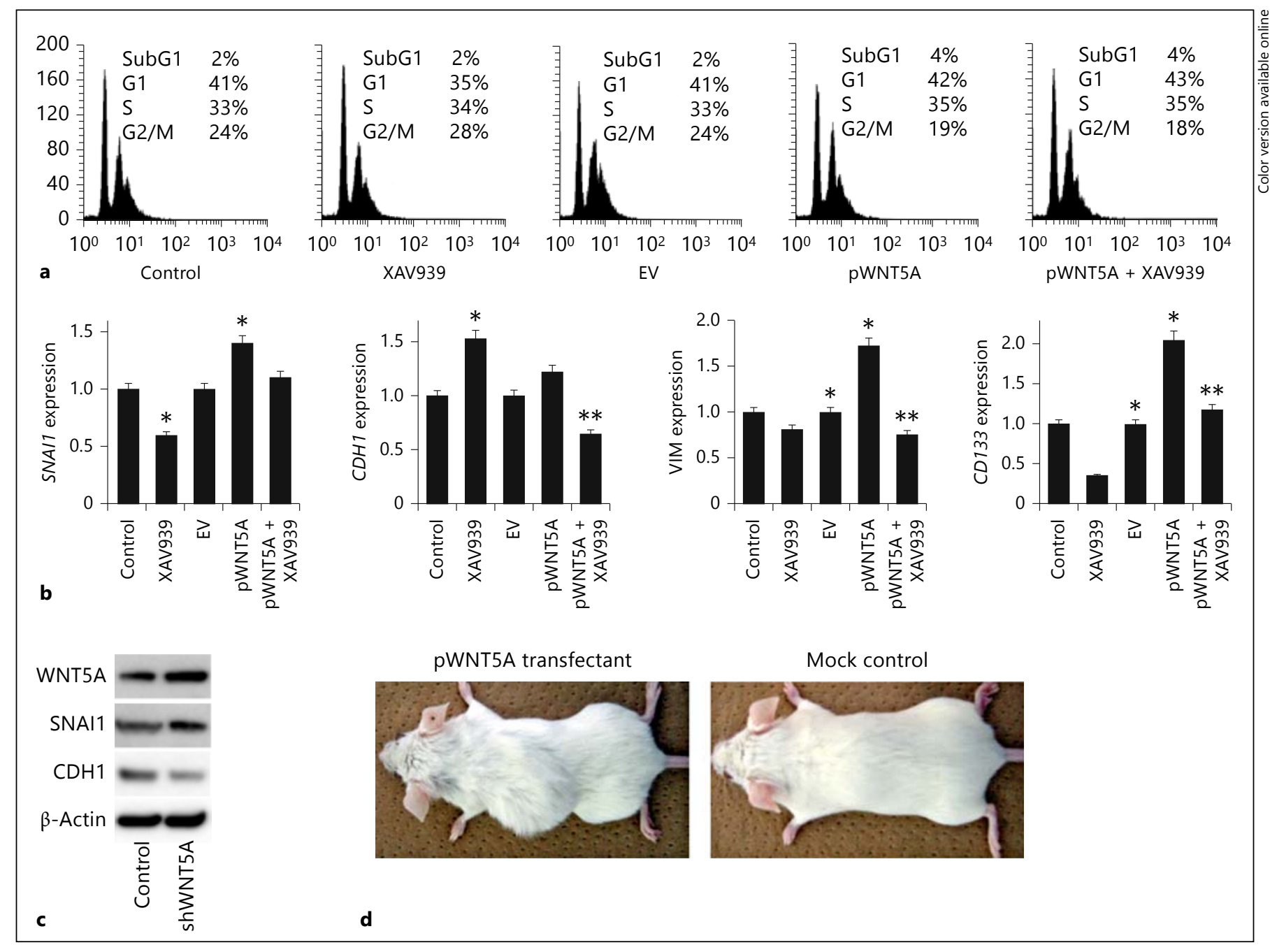

Fig. 2. WNT5A induces EMT and increases CSC marker CD133 levels in MKN-7 cells. a Results of flow cytometric analysis. Percentages of each fraction (subG1, G1, S or G2/M) are shown in the upper right corner of each fluorogram. For the suppression of WNT signaling, WNT inhibitor XAV939 was added to the culturing media. b Results of real-time RT-PCR in MKN-7 cells in the presence or absence of pWNT5A transfection and/or treatment with XAV939. The mRNA levels of GAPDH expression were analyzed as a loading control. ${ }^{*} \mathrm{p}<0.05$; ${ }^{* *} \mathrm{p}<0.01$. EV $=$ empty vector. c Expression of WNT5A, SNAI1 and CDH1 proteins in pWNT5A transfectant. $\beta$-Actin was used as a control. d Representative illustration of the tumorigenicity test. Note that subcutaneous tumor developed in the SCID mouse inoculated with pWNT5A-transfected MKN-7 cells. absence of WNT activity. Prior to the experiments, we confirmed that gene transduction and treatment with XAV939 WNT inhibitor did not influence cell viability. pWNT5A transduction did not alter the fraction of cell cycle (subG1, G1, S and G2/M phases) in the presence or absence of XAV939 (fig. 2a). Transfection of pWNT5A increased the mRNA levels of the EMT transcription factor SNAI1, mesenchymal marker VIM and CSC marker CD133 (fig. 2b). Although downregulation of the epithelial cell marker $C D H 1$ was not significant, the data sug-

WNT5A Regulates EMT and CSCs in Human Gastric Carcinoma Cells gested the possible role of WNT5A in the induction of EMT and the restoration of CSC properties. At the protein level, increased levels of WNT5A and SNAI1 were detected as well as a slight decrease in CDH1 levels (fig. 2c). We also attempted to evaluate VIM and CD133 at the protein level; however, endogenous expression of VIM and CD133 was not detected by Western blot (data not shown). To further confirm this result, we analyzed the effect of WNT5A on tumorigenicity in SCID mice. Using a limiting dilution assay, MKN-7 cells were inocu- 


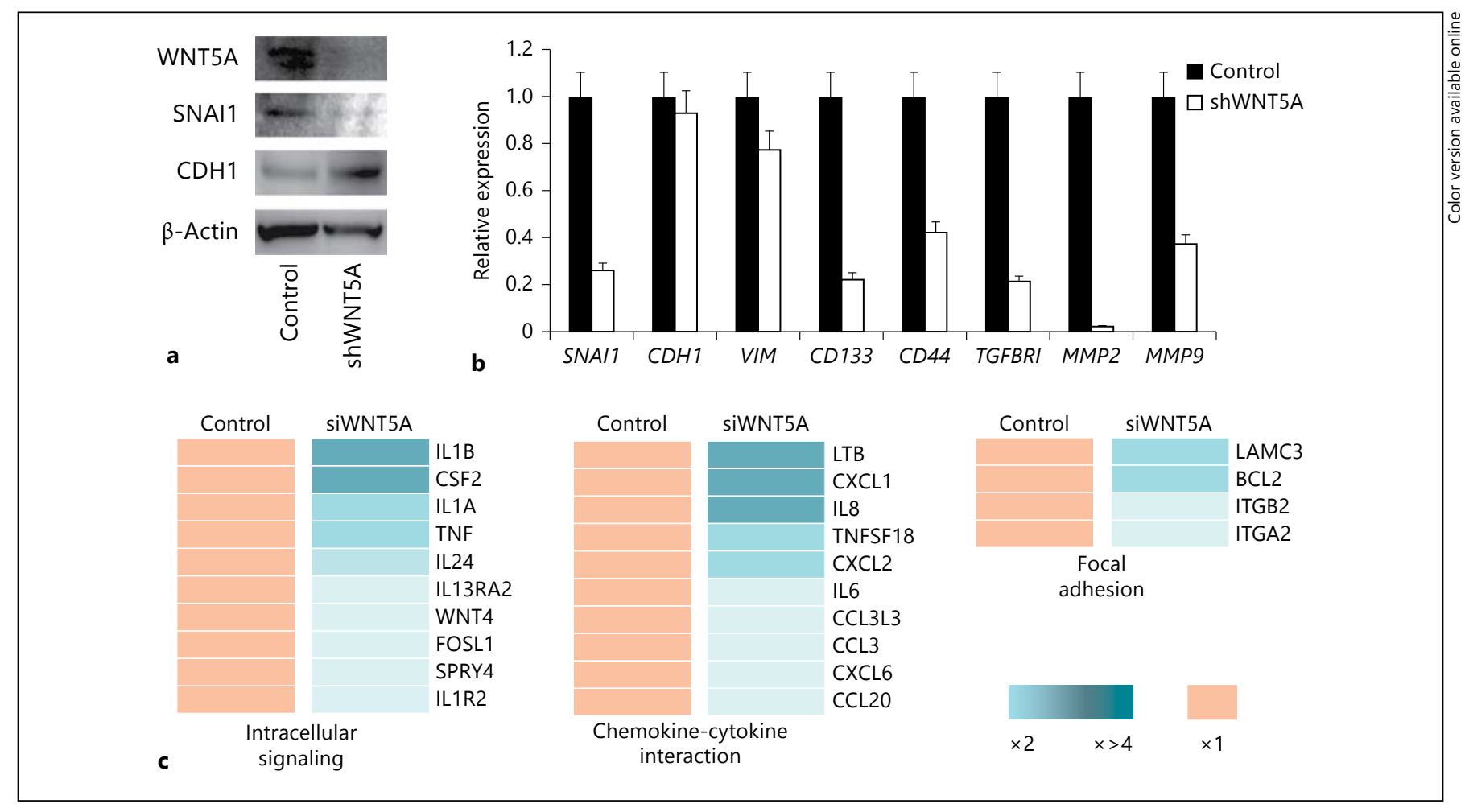

Fig. 3. Knockdown of WNT5A suppresses gene expression related to the EMT and CSCs. a Knockdown of WNT5A was performed using a lentiviral shRNA transduction system. Altered levels of WNT5A and EMT-related proteins were detected by Western blot. b Expression of EMT- and CSC-related genes in

Table 1. Tumorigenicity of MKN-7 cells

\begin{tabular}{lllllr}
\hline $\begin{array}{l}\text { Cells per } \\
\text { injection }\end{array}$ & pWNT5A & & & EV & \\
\cline { 2 - 3 } \cline { 5 - 6 } & incidence & $\%$ & & incidence & $\%$ \\
\hline $1 \times 10^{5}$ & $4 / 4$ & 100 & & $4 / 4$ & 100 \\
$1 \times 10^{4}$ & $4 / 4$ & 100 & & $2 / 4$ & 50 \\
$1 \times 10^{3}$ & $3 / 4$ & 100 & & $2 / 4$ & 50 \\
Frequency $^{\mathrm{a}}$ & $3.25 \times 10^{3}$ & & & $5.05 \times 10^{4}$ & \\
\hline
\end{tabular}

Incidence represents number of tumors/number of injections. $\mathrm{EV}=$ empty vector. ${ }^{\mathrm{a}}$ The initiating cell frequency was calculated using extreme limiting dilution analysis software (http://bioinf. wehi.edu.au/software/elda/).

lated into SCID mice subcutaneously in the presence or absence of pWNT5A (fig. 2d). As summarized in table 1, the tumorigenicity of the pWNT5A transfectant was significantly upregulated in comparison with the mock control $(\mathrm{p}<0.05)$.
shWNT5A-infected MKN-7 cells. The mRNA levels of GAPDH expression were analyzed as a loading control. c Results of a cDNA microarray analysis of gene expression involved in intracellular signaling, chemokine-cytokine interaction and focal adhesion.

\section{Knockdown of WNT5A Inhibits WNT Signaling}

Next, we established shWNT5A-transfected MKN-7 cells to develop the results obtained above (fig. 3a). Suppression of endogenous WNT5A expression effectively decreased the mRNA and protein levels of SNAI1 and CD133, whereas altered expression of CDH1 and VIM was not significant (fig. 4). At the mRNA level, the CSC marker CD44 was also downregulated, similarly to TGFBRI, MMP2 and MMP9. Furthermore, we attempted a cDNA microarray to comprehensively identify the genes differentially influenced by WNT5A. In MKN-7 cells, depletion of WNT5A induced downregulation of genes involved in intracellular signaling (IL1A/B and WNT4), chemokine-cytokine interaction (CXCL1/2/6) and focal adhesion (LAMC3 and ITGA2/B2).

\section{High Expression of WNT5A at the Invasive Front}

Cancer-Stromal Border

A previous study demonstrated that WNT5A is required for the migration and invasive ability of gastric can- 

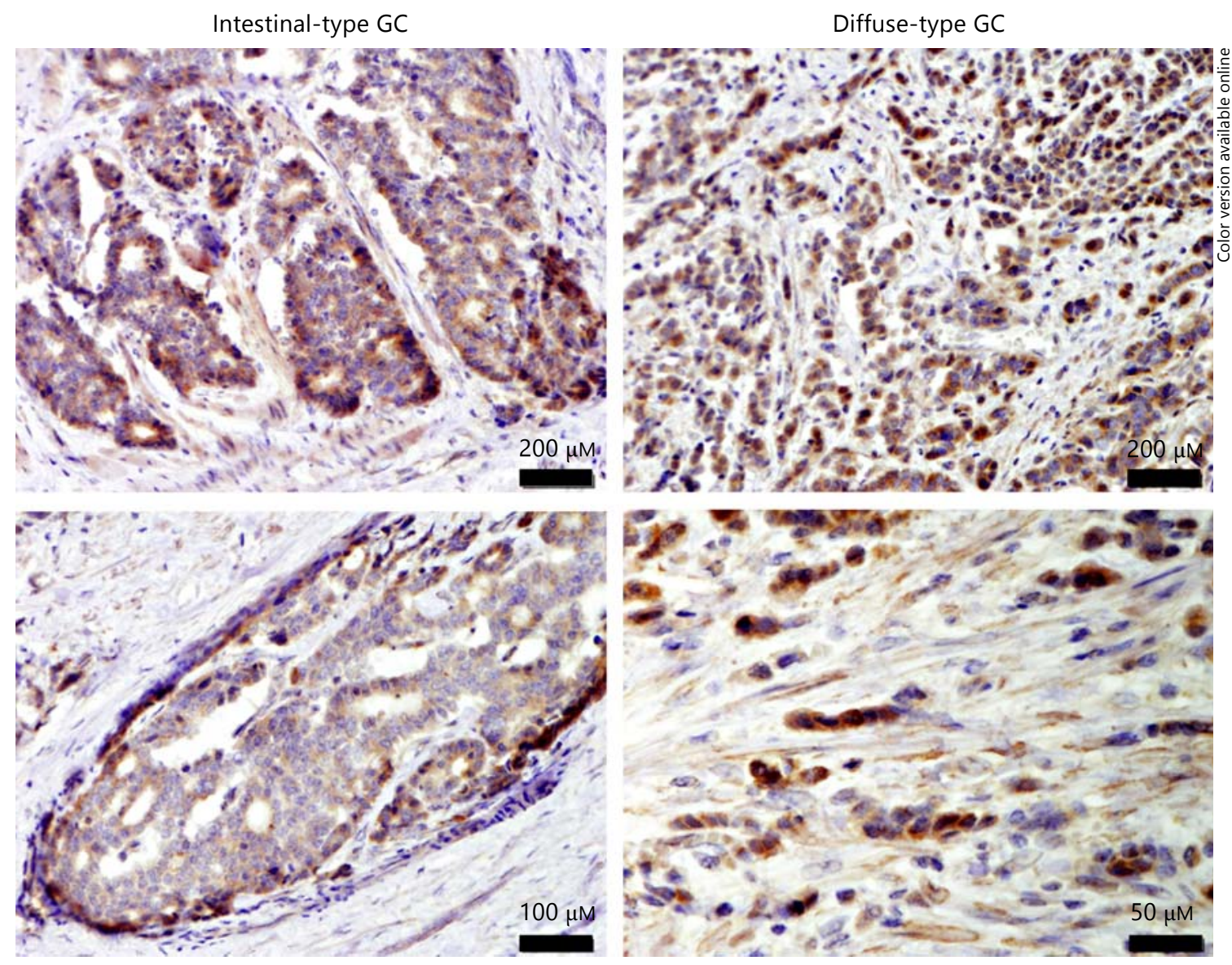

Fig. 4. Representative illustrations of immunohistochemistry of WNT5A in human GC tissues (intestinal- and diffuse-type GC cases). Note that high expression of WNT5A was detected in the peripheral site of cancer nests in intestinal-type GC. In diffuse-type GC, high WNT5A expression was detected homogenously.

cer cells and that the expression of WNT5A is correlated with aggressiveness and poor prognosis of gastric cancer [6]. Among a total $42 \mathrm{GC}$ cases, high levels of WNT5A expression were observed in 29 (66\%; fig. 4). The WNT5A status was not statistically correlated with gender, age, depth of invasion or incidence of lymph node metastasis; however, WNT5A expression was more frequently detected in diffuse-type GC cases than in intestinal-type GC cases $(p<0.041$; table 2). Interestingly, at the invasive front of intestinal-type GC cases, WNT5A was expressed especially in the periphery of the tumor nest (fig. 4).

\section{Discussion}

Activation of Frizzled receptors by WNT ligands disrupts the ademonatous polyposis coli (APC)- $\beta$-catenin complex and results in the translocation of $\beta$-catenin to the nucleus, where it associates with the Tcf/Lef transcription factor to activate specific WNT target genes $[30,31]$. Recent studies have documented that WNT signaling is crucially important in maintaining stemness in normal tissue stem cells and is a common pathway that is deregulated in human GCs [32]. Indeed, expression of WNT5A was found in all GC cell lines analyzed in this study. Although there were variations in each cell line, the expression of WNT5A is likely to be common in GC cells. Supporting this, high levels of WNT5A expression have been frequently detected in human GC tissues. Activation of WNT signaling by WNT5A may be one of the common events in human gastric carcinogenesis. Also, overexpression of WNT5A downregulated cell proliferative activity in vitro and promoted subcutaneous tumorigenesis in the mouse xenograft in vivo. The results obtained in the experiments with pWNT5A transfection into MKN-7 cells were
WNT5A Regulates EMT and CSCs in Human Gastric Carcinoma Cells
Pathobiology 2013;80:235-244 DOI: $10.1159 / 000346843$ 
Table 2. Relationship between WNT5A expression and clinicopathologic findings in human GC

\begin{tabular}{|c|c|c|c|c|c|}
\hline & \multicolumn{4}{|c|}{ WNT5A expression } & \multirow{3}{*}{$\begin{array}{l}\mathrm{p} \\
\text { value }\end{array}$} \\
\hline & \multicolumn{2}{|c|}{ positive } & \multicolumn{2}{|c|}{ negative } & \\
\hline & $\mathrm{n}$ & $\%$ & $\mathrm{n}$ & $\%$ & \\
\hline Gender & & & & & 0.620 \\
\hline Male & 20 & 43 & 10 & 29 & \\
\hline Female & 9 & 21 & 3 & 7 & \\
\hline Age & & & & & 0.227 \\
\hline$>65$ years & 18 & 38 & 10 & 21 & \\
\hline$<65$ years & 11 & 31 & 3 & 14 & \\
\hline Subtype & & & & & 0.041 \\
\hline Intestinal type & 10 & 24 & 9 & 21 & \\
\hline Diffuse type & 19 & 45 & 4 & 10 & \\
\hline Depth of invasion ${ }^{b}$ & & & & & 0.469 \\
\hline $\mathrm{T} 1 / \mathrm{T} 2$ & 6 & 14 & 6 & 14 & \\
\hline T3/T4 & 23 & 55 & 7 & 17 & \\
\hline Lymph node metastasis & & & & & 0.395 \\
\hline Positive & 18 & 43 & 3 & 7 & \\
\hline Negative & 11 & 26 & 10 & 24 & \\
\hline
\end{tabular}

$\mathrm{p}$ values less than 0.05 were considered to be statistically significant.

${ }^{\text {a }}$ Histological types were classified into 2 groups according to whether the tumor cells showed tubular formation (intestinal type) or diffuse infiltration (diffuse type).

${ }^{c}$ Depth of invasion was classified according to the TNM classification of the International Union Against Cancer.

compatible with the idea that WNT5A might be a key regulator for the acquisition of CSC properties. WNT signaling activity is a marker for colon CSCs and is regulated by myofibroblast-secreted hepatocyte growth factor in nearby colon cancer cells [31]. This may also support our conclusion that the stemness of GC cells is orchestrated by WNT5A.

Morphologically, the pWNT5A-transfected MKN-7 cells had a spindle shape and showed high cell motility. Exogenous WNT5A expression effectively increased the levels of SNAI1, a key transcription factor for EMT [33]. Subsequent downregulation of $\mathrm{CDH} 1$ and upregulation of VIM was also detected, which was suppressed by treatment with the XAV939 WNT inhibitor. Unregulated activation of WNT5A has been associated with invasiveness and several tumor types. In a study of Dissanayake et al. [34], their data strongly implicated WNT5A as an effector of EMT in melanoma cells via upregulation of SNAI1 and downregulation of CDH1. Although the WNT5A status did not correlate with the depth of invasion or the incidence of lymph node metastasis in human
GC tissues, the suppression of WNT5A decreased TGFBI and matrix metalloproteinases (MMPs). TGF- $\beta$ has been documented to promote CSC and induce EMT; TGF- $\beta$ is necessary for glioma-initiating cell self-renewal and tumorigenicity in vitro and in vivo and is associated with frequent expression of TGFBRI in diffuse-type GCs [35]. Therefore, high TGFBRI expression may increase the sensitivity to TGF- $\beta$. Moreover, in MKN-7 cells, the interference of endogenous WNT5A decreased MMP2 and MMP9, suggesting that WNT5A may control cell invasive activity. Further investigations will be required for the control of cancer infiltration and progression by WNT5A.

In the present study, WNT5A expression was frequently detected in human GC cases. Kurayoshi et al. [6] demonstrated that WNT5A stimulates cell migration by regulating focal adhesion complexes in GC cells and close correlation between WNT5A status and aggressiveness in human GC tissues. We think the discordance with our results may be caused by the specificity of antiWNT5A antibody; they generated anti-WNT5A antibody by immunization with synthetic peptides corresponding to residues 275-290 of human WNT5A that react with WNT5A only, whereas the anti-WNT5A antibody generated by immunization with synthetic peptides corresponding to residues 165-181 of human WNT5A recognizes WNT5A and WNT5B [6]. In this study, we should have excluded the possible cross-reactivity of anti-WNT5A antibody with human WNT5B. Of note, at the invasive front of intestinal-type GC cases, $\mathrm{WN}$ T5A was expressed in the periphery of the tumor nest. According to the results of the gene transduction experiments, high WNT5A expression possibly induces EMT in GC cells with increased levels of SNAI1 expression. In our previous study, in a three-dimensional culture of SW480 colon cancer cells, treatment with TGF- $\beta$ caused SNAI1 expression to be scattered on the surface of the tumor sphere [36]. Moreover, transduction of pSNAI1 into CD133- SW480 cells effectively upregulated CD133+ cells with high tumorigenic potential in the mouse xenograft model, suggesting that the SNAI1-mediated EMT may overlap with the acquisition and maintenance of CSC properties in GC cells $[36,37]$. These findings indicate the possible effect of the tumor microenvironment, including CAFs, on the induction of EMT and subsequent gain of CSC properties by cancer-stromal interaction. Understanding the tumor microenvironment may lead to elucidation of the relationship between the induction of EMT and the gain of CSC properties. 
Using a cDNA microarray, we identified several genes that may be downstream targets within WNT signaling. Inactivation of WNT5A was associated with WNT4 expression. Wnt4 stimulation was shown to activate the small GTPase Racl and JNK kinase in a human hematopoietic cell line and contribute to murine hematopoietic cell proliferation. Moreover, WNT5Amediated activation of WNT signaling was related to interleukins and to several angiogenic cytokines in the CXCL family, which may contribute to the promotion of angiogenesis. In colorectal cancer, it has been documented that interleukin- 6 increased the numbers of
CSCs and promoted tumor formation in vitro and in vivo. The interleukin-6-mediated increase of CSCs may be directly connected to tumor progression and metastasis.

In conclusion, we confirmed the significant role of autocrine WNT5A in the construction and maintenance of an advantageous tumor microenvironment for the restoration and maintenance of CSCs. WNT5A may be a useful marker for CSCs in human GC tissues, and understanding the function of WNT5A may lead to the development of new chemotherapeutic agents for human GC.

\section{References}

$\checkmark 1$ Cunha GR, Hayward SW, Wang YZ, Ricke 10 Grudzien P, Lo S, Albain KS, Robinson P, RaWA: Role of the stromal microenvironment in carcinogenesis of the prostate. Int J Cancer 2003; 107:1-10.

2 Olumi AF, Grossfeld GD, Hayward SW, Carroll PR, Tlsty TD, Cunha GR: Carcinoma-associated fibroblasts direct tumor progression of initiated human prostatic epithelium. Cancer Res 1999;59:5002-5011.

-3 Nishimura K, Semba S, Aoyagi K, Sasaki H, Yokozaki H: Mesenchymal stem cells provide an advantageous tumor microenvironment for the restoration of cancer stem cells. Pathobiology 2012;79:290-306.

4 Logan CY, Nusse R: The Wnt signaling pathway in development and disease. Annu Rev Cell Dev Biol 2004;20:781-810.

5 Nishita M, Yoo SK, Nomachi A, Kani S, Sougawa N, Ohta Y, Takada S, Kikuchi A, Minami Y: Filopodia formation mediated by receptor tyrosine kinase Ror2 is required for $\mathrm{Wn}$ t5a-induced cell migration. J Cell Biol 2006; 175:555-562.

6 Kurayoshi M, Oue N, Yamamoto H, Kishida M, Inoue A, Asahara T, Yasui W, Kikuchi A: Expression of Wnt-5a is correlated with aggressiveness of gastric cancer by stimulating cell migration and invasion. Cancer Res 2006; 66:10439-10448.

7 Enomoto M, Hayakawa S, Itsukushima S, Ren DY, Matsuo M, Tamada K, Oneyama C, Okada M, Takumi T, Nishita M, Minami Y: Autonomous regulation of osteosarcoma cell invasiveness by $\mathrm{Wnt} 5 \mathrm{a} / \mathrm{Ror} 2$ signaling. Oncogene 2009;28:3197-3208.

$\checkmark 8$ Yamamoto H, Kitadai Y, Oue N, Ohdan H, Yasui W, Kikuchi A: Laminin gamma2 mediates Wnt5a-induced invasion of gastric cancer cells. Gastroenterology 2009; 137:242252.

9 Ann EJ, Kim HY, Seo MS, Mo JS, Kim MY, Yoon JH, Ahn JS, Park HS: Wnt5a controls Notch1 signaling through CaMKII mediated degradation of the SMRT corepressor protein. J Biol Chem 2012;287:36814-36829. jan P, Strack PR, Golde TE, Miele L, Foreman KE: Inhibition of Notch signaling reduces the stem-like population of breast cancer cells and prevents mammosphere formation. Anticancer Res 2010;30:3853-3867.

11 Singh A, Settleman J: EMT, cancer stem cells and drug resistance: an emerging axis of evil in the war on cancer. Oncogene 2010;29:47414751.

12 Campbell LL, Polyak K: Breast tumor heterogeneity: cancer stem cell or clonal evolution? Cell Cycle 2007;6:2332-2338.

13 Clarke MF, Fuller M: Stem cells and cancer: two faces of eve. Cell 2006;124:1111-1115.

14 Polyak K, Hahn WC: Roots and stems: stem cells in cancer. Nat Med 2006;12:296-300.

15 SinghSK, Hawkins C, ClarkeID, Squire JA, Bayani J, Hide T, Henkelman RM, Cusimano MD, Dirks PB:Identification of humanbrain tumour initiating cells. Nature 2004;432:396-401.

16 O'Brien CA, Pollett A, Gallinger S, Dick JE: A human colon cancer cell capable of initiating tumour growth in immunodeficient mice. Nature 2007;445:106-110.

17 Ma S, Chan KW, Hu L, Lee TK, Wo JY, Ng IO, Zheng BJ, Guan XY: Identification and characterization of tumorigenic liver cancer stem/ progenitor cells. Gastroenterology 2007;132: 2542-2556.

18 Olempska M, Eisenach PA, Ammerpohl O, Ungefroren H, Fandrich F, Kalthoff H: Detection of tumor stem cell markers in pancreatic carcinoma cell lines. Hepatobiliary Pancreat Dis Int 2007;6:92-97.

19 Vander Griend DJ, Karthaus WL, Dalrymple S, Meeker A, DeMarzo AM, Isaacs JT: The role of CD133 in normal human prostate stem cells and malignant cancer-initiating cells.

20 Yin AH, Miraglia S, Zanjani ED, Almeida-Porada G, Ogawa M, Leary AG, Olweus J, Kearney J, Buck DW: AC133, a novel marker for human hematopoietic stem and progenitor cells. Blood 1997;90:5002-5012. Cancer Res 2008;68:9703-9711.
21 Bidlingmaier S, Zhu X, Liu B: The utility and limitations of glycosylated human CD133 epitopes in defining cancer stem cells. J Mol Med 2008;86:1025-1032.

22 Hojo H: Establishment of cultured cell lines of human stomach cancer-origin and their morphological characteristics (in Japanese). Niigata Igakukai Zassi 1977;91:737-752.

23 Motoyama T, Hojo H, Watanabe H: Comparison of seven cell lines derived from human gastric carcinomas. Acta Pathol Jpn 1986;36: 65-83.

24 Yanagihara K, Ito A, Toge T, Numoto M: Antiproliferative effects of isoflavones on human cancer cell lines established from the gastrointestinal tract. Cancer Res 1993;53:5815-5821.

25 Yanagihara K, Seyama T, Tsumuraya M, Kamada N, Yokoro K: Establishment and characterization of human signet ring cell gastric carcinoma cell lines with amplification of the cmyc oncogene. Cancer Res 1991;51:381-386.

26 Ochiai A, Yasui W, Tahara E: Growth-promoting effect of gastrin on human gastric carcinoma cell line TMK-1. Jpn J Cancer Res 1985;76:1064-1071.

27 Japanese Gastric Cancer Association: Japanese Classification of Gastric Carcinoma - 2nd English Edition. Gastric Cancer 1998;1:10-24.

28 Vaiopoulos AG, Kostakis ID, Koutsilieris M, Papavassiliou AG: Colon cancer stem cells. Stem Cells 2012;30:363-371.

29 Brablets S, Schmalhofer O, Brablets T: Gastrointestinal stem cells in development and cancer. J Pathol 2009;217:307-317.

- 30 Tetsu O, McCormick F: $\beta$-catenin regulates expression of cyclin D1 in colon carcinoma cells. Nature 1999;398:422-426.

31 Vermeulen L, De Sousa E, Melo F, van der Heijden M, Cameron K, de Jong JH, Borovski T, Tuynman JB, Todaro M, Merz C, Rodermond H, Sprick MR, Kemper K, Richel DJ, Stassi G, Medema JP: Wnt activity defines colon cancer stem cells and is regulated by the microenvironment. Nat Cell Biol 2010;12: 468-476.
WNT5A Regulates EMT and CSCs in Human Gastric Carcinoma Cells 
-32 Voon DC, Wang H, Koo JK, Nguyen TA, Hor YT, Chu YS, Ito K, Fukamachi H, Chan L, Thiery JP, Ito Y: Runx3 protects gastric epithelial cell against epithelial-mesenchymal transition-induced cellular plasticity and tumorigenicity. Stem Cells 2012;30:2088-2099.

- 33 Ikenouchi J, Matsuda M, Furse M, Tsukita S: Regulation of tight junction during the epithelium-mesenchyme transition: direct repression of gene expression of claudins/occludin by Snail. J Cell Sci 2003;116:1959-1967.
Dissanayake SK, Wade M, Johnson CE, O'Connell MP, Leotlela PD, French AD, Shah KV, Hewitt KL, Rosenthal DT, Indig FE, Jiang Y, Nickoloff BJ, Taub DD, Trent JM, Moon RT, Bittner M, Weeraratna AT: The Wnt5A/ protein kinase $\mathrm{C}$ pathway mediates motility in melanoma cells via the inhibition of metastasis suppressors and initiation of an epithelial to mesenchymal transition. J Biol Chem 2007; 282:17259-17271.

- 35 Ikushima H, Todo T, Ino Y, Takahashi M, Miyazawa M, Miyazono K: Autocrine TGF- $\beta$ signaling maintains tumorigenicity of glioma-initiating cells through Sry-related HMGbox factors. Cell Stem Cell 2009;5:504-514.
36 Yusra, Semba S, Yokozaki H: Biological significance of tumor budding at the invasive front of human colorectal carcinoma cells. Int J Oncol 2012;41:201-210.

37 Floor S, van Staveren WC, Larsimont D, Dumont JE, Maenhaut C: Cancer cells in epithelial-to-mesenchymal transition and tumorpropagating-cancer stem cells: distinct, overlapping or same populations. Oncogene 2011; 30:4609-4621. 